\title{
Günter Frankenberg Hüter der Verfassung einer Zivilgesellschaft
}

Womit kann das Bundesverfassungsgericht seine Rechtsprechung in politisch umstrittenen Fragen - etwa die Kruzifix-, Soldaten sind Mörder-, Sitzblockaden-Entscheidungen-legitimieren? Stellt legal-rational begründete Autorität eine binreichende moderne Legitimationsquelle dar? Frankenberg bezweifelt das. Er schlägt stattdessen eine Legitimationsgrundlage vor, die im zivilgesellschaftlichen Konflikt ibre Wurzeln hat.

D. Red.

\section{Unrube über Karlsrube}

Im Bild ein modernes Gebäude aus Glas und Stahl, schlicht und würdig: »eine wohlproportionierce ,Orangerica'. Das architektonische Versprechen von Transparenz, ganz im Stile einer demokratischen Republik. Der Sitz des Bundesverfassungsgerichts, kurz: BVerfG. Aus dem Off informiert uns dic sonore Stimme des öffentlichrechtlichen Kommentators, das seit langem erovartetc Urteil in der Sache X sei ergangen. Schnitt. Nun treten die Mitglieder des BVerfG - farbenprächtig wie Richterkönige, verschlossen wie Subsumtionsautomaten - vor die Augen auch der Fernsehkameras und verkünden ihr Urteil. Schnitt.

Dic interessierte Öffentlichkeit hält zwar nicht den Atcm an, doch eine erhöhte Aufmerksamkeit ist dem obersten Gericht der Republik gewiß. Seine Sprüche sind häufig für eine Überraschung gut und regelmäßig kontrovers. Diesc Öffentlichkeit, so die allcin vom Kurızeitgedächtnis informierte Mcinung, wurde aus Karlsruhe im vergangenen Jahr mehr denn je mit Kontroversen bedient. In der Tat haben dic Entscheidungen zu Sitzblockaden, Soldaten, Kruzifix und - weniger spektakulär, aber verfassungspolitisch kaum weniger folgenreich - zu Einheitswerten erheblichen Staub aufgewirbelt. ${ }^{2}$ Wer freilich cin wenig in Buch der vergleichsweise kurzen Geschichre des BVerfG und in der mittlerweile auf 92 Bände angewachsenen Entscheidungssammlung blättert, stößt auf zahlreiche Urteilc und Beschlüsse, die kaum weniger umstrittcn waren: Der Strcit um die ursprünglichen Pläne Adenaucrs zur Wiederbewaffnung der Bundesrepublik führte zu einem ersten schweren Verfassungskonflikt, in dem dic Bundesregierung dem BVerfG vorwarf, süber die Bestimmungen des Grundgesetzes hinausgegangen « zu sein und »aus eigener Machtvollkommenheit Recht (zu) setzen «,

t Raschorn, Aus einer kleinen Residenz. Zum Selbstverstandnis des Bundesverfassungsgerichts, in: Daubler/Kusel (Hg.), Verfassungsgericht und Politık (1979), 149. Boshaft die Charakterisierung der Residenz des Rechts Karlsruhe: whalb so groß wie der Zentralfriedhof von Chicago, aber doppelt so tot * (S. 160 ).

2 BVerfG NJW 1995,1114 (Sitzblockaden); BVerfG NJW 1995, 3303 (Soldaten), im Nachgang zum Beschluß der 3. Kammer des Ersten Senats BVerfG NJW 1994, 2943; BVerfG EuGRZ 1995, 359 iff (Kruzifix); BVerfG NJ 1995, 523 (Emheitswerte). Nicht zufallig verpaßte "Kruzifix nur knapp, von der Gesellschaft fur deutsche Sprache als nWorta des Jahres ausgezetehnet zu werden.

3 Bullecin der Bundesregierung Nr.198 (1952), 1729. Im Vorfeld der Entscheidung des BVerfG iber das 
und mit dem "Ende der deutschen Verfassungsjustiz " ${ }^{*}$ drohte. Bald danach sah sich das BVerfG massiven Protesten vornehmlich aus den juristischen Fakultäten gegenüber, als es mit unmißverständlicher Deutlichkeit dic These zurückwies, der Wechsel von der NS-Diktatur zur Bundesrepublik habe die Rechtsverhältnisse der ehemaligen Angehörigen des öffentlichen Dienstes unberührt gelassen. ${ }^{5}$ Wiederum die Bundesregierung nahm Anstoß an der laut Kanzler Adenauer "falschen Entscheidung «, die dessen Plan, cine "Deutschland-Fernsehen GmbH « zu gründen, Verfassungswidrigkeit bescheinigte. ${ }^{6}$ Ein regierungsnaher Kommentator belehrte das Gericht, es habe mit seinem ı. Fernseh-Urteil "Ulbricht und seinen Genossen gedicnt "?.

Die Epoche, in der das BVerfG von seinen Gegnern als "Kampfinstrument der linken Opposition tituliert werden konnte, ${ }^{8}$ ging in den $60 e r$ Jahren zu Ende, wenn sie denn je existierte. Mit dem für Grundrechtsfreunde schwer erträglichen (und verfassungsdogmatisch nicht leicht nachvollyiehbaren) Abhör-Urteil" setzte sich cine etatistische Tendenz durch. Aus Anlaß dieser Entschcidung, die der Staatsgewalt unter Modifikation »elementarer Verfassungsgrundsätze" dic Befugnis zum Abhören zusprach, trat erstmals der interne verfassungsrichterliche Dissens in einem Sondervotum zutage. ${ }^{10}$ Ebenfalls eher den Imperativen innerer Sicherheit als rechtsund verfassungsstaatlicher Logik gehorchte auch der viel kritisicrte "Radikalenbeschluß«, der den Beamten im Namen nicht der Verfassungs-, sondern der Staatstreue auferlegte, wsich jederzeit in diesem Staat zu Hause fühlen ". "

Daß Etatismus freilich nicht als Regierungsfrcundlichkeit mißzuverstchen ist, demonstrierte das BVerfG in den 7oer Jahren, als sich die sozialliberale Regierung aufmachte, längst überfällige soziale Reformen ins Werk zu setzen. Regelmäßig von der im Parlament überstimmten Opposition angerufen, korrigierte das Gcricht cine Reihe von Reformprojekten. Die sogenannte Fristenlösung scheitertc an der »objektiven Wertordnung « des Grundgesetzes, der das Gericht, konstruktiv über Art. 2 II GG, einc Schutzpflicht des Staates auch für das ungeborene Leben entnahm. ${ }^{2}$ Diese Entscheidung empörte neben der Regierungsmehrheit vor allem die außerparlamentarischen Bewegungen, die das Selbstbestimmungsrecht der Fraucn favorisierten. Wiederum die sozialliberale Regicrung war über die verfassungsrichterliche Beurteilung der Brandtschen Entspannungspolitik erbost."

Gutachten des Bundesprasidenten zur Verfassungsmaßigkeit der Europaischen Verteidigungsgemeinschaft außerte der damalige Justizminister Dehler, er hoffe, „daß sich beim BVerfG der Geist des Sozialismus niche (auswirke). . Die dann gefallte Entscheidung des Gerichts betreffend die bindende Wirkung seines noch zu erstellenden Gutachtens quittierte der Justzzminister als „vollig rechtlosa (Lıetzmann, Das Bundesverfassungsgericht, 1988, 102 f.). Dehler besiegelte damit das Ende seiner politischen Karriere. Vgl. Leicht, DIE ZEIT Nr. 35 (1955) und Wesel, DIE ZEIT Nr. 40 (1995).

4 Bulletin d. Bundesreg. Nr. 185 v. 26.11.1952.

s BVerfGE 1, 167/177f. (G 131 - Ubernahme sverdrangter « Angehoriger des offentlichen Dienstes); 3, 58 (Übernahme von Beamten); 3, 187 (Übernahme von Angestellten); 3, 288 (Ubernahme von Soldaten)im offenen Widerspruch zu BGHZ 13, 299.

6 BVerfGE 12, 20 (1. Fernseh-Urteil).

7 Wagner-Kauß, in: die politische meinung nr. 58 (1961).

$8 \mathrm{Vgl}$. Ridder, In Sachen Opposition, in: FS f. Adolf Arndt, hrsg. v. Ehmke (Frankfurt/M. 1969). Zu einer anderen Einschatzung: Rudder, Unerfulltes Grundgesetz? In: vorgange Nr. 37 (1979), 101.

9 BVerfGE 30, 1 (Abhor-Urteil).

10 BVerfGE 30, 33 (Sondervotum zum Abhor-Urtell).

„1 BVerfGE 39, 334 (\$Radikalen $\alpha-B e s c h l u ß)$. Zur Bandbreite der Kritik vgl. Bockenforde, Verhaltensgewahr oder Gesinnungstreue?, in: ders., Staat, Verfassung, Demokratie (1991), 277 ff.; Abendroth, Das Bundesverfassungsgericht und die Berufsverbote im offentlichen Dienst, in: ders., Arbeterklasse, Staat und Verfassung, 295 ff.; Schlink, Der Staat 1976, 33 fff.; Denninger, Verfassungstreue und Schutz der Verfassung, VVDStRL 37 (1979), 7 ff.

$12 \mathrm{BVerfGE} 39,1$ und 88,203 ( $\$ 218 \mathrm{StGB} /$ Schwangerschaftsabhruch).

13 BVerfGE 35, 257 (Grundlagen-Vertrag). Aus dem Kabınett wird die wentg galante Außerung eınes Mitglıedes der Bundesregierung kolportiert, man werde sıch von den sacht Arschlochern in Karlsruhe nicht wdie Ostpolıtik kaputtmachene lassen. Zit. nach Leicht, DIE ZEIT (Fn. 3). 
Unübersichtlicher wird die Lage in den 8oer Jahren. Die Freude der Christdemokraten am Verfassungsgericht crhielt, bald nachdem diese die Kanzlermehrheit stellten, durch die Urteile zur Demonstrationsfreiheit ${ }^{14}$ und zum Volkszählungsgesetz von $1983^{13}$ erhebliche Dämpfer. Ein Trost für diese, daß das BVerfG, nachdem es 1978 die sozialliberale Wehrpflichtnovelle kassiert hatte, ${ }^{16}$ nunmehr die von Christ- und Freidemokraten ersonnene "lästige Alternative" des Zivildienstes absegnete und dabei das Kunststück fertigbrachte, dessen gegenüber dem Wehrdienst drei Monate längere Dauer mit dem an sich klaren, entgegenstehenden Wortlaut von Art. I 2a II 2 GG zu versöhnen. ${ }^{17}$ Ein weiterer Trost für Staatsfreunde: Die Protestform des zivilen Ungehorsams fand in der ersten Mutlangen-Entscheidung zwar Erwähnung, aber im Rahmen von $\$ 240$ StGB etwa als Grund, die Verwerflichkeit der Nötigung zu verneinen, keine Gnade. ${ }^{18}$ Obwohl sich die knappe $4: 4$-Entscheidung bald zu festigen schien, ${ }^{19}$ überraschte der Erste Senat schließlich Publikum und Fachgerichte mit einem soverruling und befreite diesc Form des symbolischen Protests immerhin vom Odium der Nötigung. ${ }^{20}$ Und brachte damit - und mit der 1995 wiederholten Beantivortung der Frage, ob Soldaten unter dem Schutz von Art. s I GG unter Umständen Mörder genannt werden dürfen ${ }^{21}$ - die durch eine seit insbesondere der Lïth-Entscheidung ${ }^{22}$ verhältnismäßig konsequente, vermeintlich allzu libcrale ${ }^{23}$ Rechtsprechung ohnehin gereizten Staatsfrcunde gegen sich auf. ${ }^{24}$

Die Kritik am BVerfG, so die Bilanz, wechselt von Fall zu Fall die Seite. ${ }^{25} \mathrm{Da}$ das Gericht, streng genommen, keine Vergleiche schließt, kennt jede seiner Entscheidungen Sieger und Verliercr, denen die Nicderlage nahelegt, ihre Enttäuschung durch Urteilsschelte kleinzuarbeiten. Solcher durchweg am Ergebnis orientierten Kritik entgeht das Gericht auch nicht dadurch, daß es unter der Flagge "praktischer Konkordanz«Kompromisse sucht ${ }^{26}$, mit »verfassungskonformen Auslegungen« dem

${ }_{14}$ BVerfGE 69, 3 Is (Brokdorf). Insbesondere die Verpflichtung der Sicherheitskrafte zu versanmmlung5freundlıchem Verhalten (sog. Kooperationsprınzip) sueß in Kreisen der Exckutive auf wenig Gegenliebe.

is BVerfGE 65, I (Volkszahlung).

16 BVerfGE 48,127 (Wehrpflicht I).

17 BVerfGE 69, 1 (Verlangerung des Zivildienstes).

: 8 BVerfGE 73, 206 (Sitzblockade Mutlangen I). Die Stimmengleıchheit im Ersten Senat fuhrte gem. \$ is III 3 BVerfGG dazu, daß en Verfassungsversto $B$ nicht festgestellt werden konnte.

19 Weitere Entscheidungen zu Sitzblockaden: BVerfGE 76, 21 (Sitzblockade Neu-Ulm); BVerfG NStZ 1991, 279, NJW 1991, 971 und NJW 1992, 2688 (Aufrufc zu Sitzblockaden).

20 BVerfG NJW 1995, 1114 (Sitzblockade Großengstingen). Dreh- und Angelpunkt dieses Meinungswandels auf der Basis veranderter Mehrhettsverhaltnisse um Ersten Senat ist freilich dic Unbestimmtheit des "vergeistigten Gewaltbegriffs" im Sinne von Art. 103 II GG. Vgl. hicrzu auch BVerfGE 87, 406.

21 BVerfG NJW 1994, 2943 und BVerfG NJW 1995, 3303

22 BVerfGE. 7, 198 (Boykottaufruf); vgl. auch E 25,256 (Blinkfuer). Auch dic Luth-Entscheidung war seincrzert kaum weniger kontrovers als heutige Spruche zu Art. s I GG, die als zu liberal gescholten werden. Es entbehr nicht einer gewissen Ironie, wenn F. K. Fromme, die Staatskassandra voni Main, der in der Luth-Entscheıdung fundierten Dogmarik zur Meınungsfreiheit, in Milde zuruckblickend, eıne gewisse zeitgeschichtliche Berechtigung nicht versagen will, aber im gleichen Atemzug und Argument doch dazu auffordert, den Schutzbereich von Art.5 I GG in einer konsolidierten, vom Schatten der Vergangenheit befreiten Demokratie etnzuengen.

23 So Schmitt Glaeser, JZ 1983, $95 \mathrm{ff}$; Wurtenberger, NJW $1983,1144 \mathrm{ff}$; Kricle NJW 1994, $1897 \mathrm{ff}$.

24 Nicht immer laufen deren Argumente so aus dem Ruder wie Roclleckes Dictum: -Sitzblockaden sind

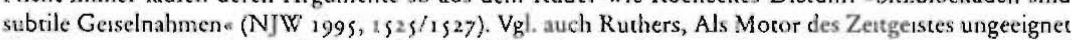
(FAZ v. 9.6.1995, 10), der dem BVerfG vorwirft, es beuge sich dem Zeitgeist. Den Soldaten-Beschluß halt er fur "ein seltsames Gemisch aus Schizophrenie und systematischer Heucheles" (FAZ v. 22. 5. I995). Reifenrarh, Wider den Zetrgeist, (FR v. 29. 11. 1995), 12 behauptet mit guten Grunden das Gegenteil. So erschent der Zett-, im Unterschied zum Weltgetst wohl cine Frage der Perspektive.

2s Was nicht nur fur die Parteien verfassungsrechelicher Streitigkeıten, sondern auch fur emige professıonelle Beobachter gilt.

26 Gute Beispiele fur die methodische Angreifbarkeit der Abwagung nach Maßgabe spraktischer Konkordanze sind die Entscheidungen zur Paradoxie einer *christhchen Gemeinschaftsschule - BVerfGE ${ }_{4} 1,29$ (Baden-Wurtt); 41,65 (Bayern); 41,88 (Nordrhein-Westf.) - und zum Schulgebet (BVerfGI: 52, 223; vgl dazu Bockenforde, DOV 1980,323). 
offenen Konflikt mit dem Gesetzgeber ausweicht oder mit "Schlüsselbegriffen ${ }^{27}$ wic vor allem der Verhältnismäßigkeit ${ }^{28}$ operiert, dic einer Entscheidung die unerbittliche Schärfe nehmen (sollen). Denn unabhängig von solchen Strategien wird das BVerfG stets als zu streng oder \%u nachgiebig, seine Entschcidung als zu politisch oder zu juristisch etc. kritisiert. ${ }^{29}$

\section{Der Kruzifix-Konflikt}

Der vom doppelt falsch sogenannten "Kruzifix-Urteil « ${ }^{30}$ losgetretene Streit verläßt, insofern vergleichbar dem Verfassungskonflikt um dic Wiederbewaffnung, die Bahnen vorhersehbarer und routinierter Urteilsschelte. Damals, ein knappes Jahr nach der Konstituierung des BVerfG, wic im Sommer 1995 schlägr die Kritik am Ergebnis um in wütende Angriffe auf die Institution Verfassungsgerichtsbarkeit. Damals wie heute, auch das dürfte keine zufällige Parallele scin, droht die Exekutive mit Boykott bzw. praktiziert solchen. ${ }^{\prime \prime}$ Hemmungsloser freilich als seinerzeit die Mitglieder der Adenauer-Regierung laufen dieser Tage die Gegner des Kruzifix-Beschlusses Sturm bis Amok.

Sommer und Herbst 1995: Kein Tag vergeht, an dem nicht ein weiterer kirchlicher Würden-oder politischer Bedenkenträger, je nach Temperament, sein Unverständnis oder Entsetzen bekundet und, je nach Verfassungstrcue, scinen Protest äußert oder zum Widerstand aufruft. ${ }^{32}$ Die Entschcidung über das Kreuz im Klassenzimmer entzweit die Republik. Dem Kanzler ist sie schlicht unverständlich. Andere sehen in ihr eine Erpressung der Mchrheit der deutschen Bevölkerung, eine "Gefahr für den Fortbestand der sozialen Marktwirtschatt '13, wenn nicht gar den Untergang des christlichen Abendlandes, zumindest aber dic Umrisse einer "kreuzeslosen abendländischen Gesellschaft «34. Bayerns ehemaliger Kultusminister Hans Maier, der Vater des vom BVerf $G$ als verfassungswidrig gerügten $\mathbb{1}_{1_{3}} \mathrm{I}_{3}$ der Bayerischen Volksschulordnung (»In jedem Klassenzimmer ist ein Kreuz anzubringen. $*$ ), und sein Glaubensbruder, der Kardinal und Erzbischof von Frcising und München, stellten die Tat (gottlob nicht die Motive) der Senatsmchrheit gar auf eine Stufe mit den atheistischen Bilderstürmern des Nazi-Regimes. Und der um schiefe Metaphern selten verlegene Finanzminister Waigel fragtc die Richter, sob sie mit dieser Entscheidung nicht auch Hand an die Wurzel ihres eigenen Gerichts gelegt «ätten."3s

27 Vgl. Denninger, Verfassungsrechtliche Schlusselbegriffe, in: ders., Der gebandigte Leviathan, $158 \mathrm{ff}$.

28 Ein pragnantes Beispel: BVerfGE 50, 290 (Mitbesumnung) und aus newerer Zet BVerfC EuGRZ 1994, 245 ff. (Cannabis).

29 Nicht selten trifit solche in der Zusammenschau gegensatzlıche Krituk ein und dieselbe Entscheidung.

30 Weder handelt es sich technisch um ein Urteil (vielmehr um einen Beschluß), noch geht es nur um das Kruzifix (vielmehr auch um das schlichte Kreuz ohne Darstellung des Gekreuzigten).

31 Angesichts des laxen Sprachgebrauchs in den lezten Monaten (vgl. auch Ross, unten Fn. 35) ist darauf hinzuweısen, daß sıch die Opposition der Bayerıschen Staatsregierung gegen den Karlsruher Beschluß nucht als ziviler Ungehorsam adeln laßs. Der von ihr inituerte Gesetzesbeschluß im Bayerischen Landtag ist maskierter Rechtsungehorsam.

32 Von den zahllosen Berichten und Kommentaren siehe nur Knapp, Die Frau in der Zentrale kriegt den ersten Arger ab, FR v. 16.8. 1995; Jungel, Die unsichtbare Kirche, FAZ v. 14.9. 1995; Schostack, Die Preußen sitzen jetzt in Karlsruhe, FAZ v. 25.9. 1995; Gunther, Das Recht, die Moral und die Mehrhert, FR v. 22.8. 1995 und Wehler, Der Kampf gegen Karlsruhe, in: DIE ZEIT Nr. 49 (i995).

33 So allen Ernstes der Bischof von Hildesheim, FAZ v. 25.8. 1995 .

34 Kardinal Meisner, Bericht der FR v, 12.8. 1995.

35 FR v. 17.8. und 9.9.1995. Zur aufs Ganze gehenden, auf die Insutution der Verfassungsgerıchtsbarkeıt zielenden Kritik vgl. die Erklarung von Justizminıstern der SPD und des Bundnıs 9o/Dic Grunen, FAZ v. 25. 8. 1995 . Vgl. außerdem Augstein, Hande weg rom Gericht, Der Spiegel Nr. 35 (1995), 36 (eine wirre Attacke auf das und zuglech eine Verteidigung des BVerfG); Ross, Huter der Verfassung, FAZ v. 
Die Angegriffenen zeigten Wirkung und Abwehrbereitschaft. Anfangs wies der Vorsitzende des Ersten Senats die Verantwortung für den Streit urns Kreuz den starrsinnigen bayerischen Kultusbehörden zu. ${ }^{16}$ Dann besserte er, verfahrensrechtlich nicht ganz korrekt, in seiner Pressemitteilung den in der Tat mißverständlichen Ersten Leitsatz nach. ${ }^{37}$ Auf dem Spiel stehe mittler weilc neben der Autorität des Gcrichts auch dic Verbindlichkeit des Rechts, sekundierte sein Richterkollege Grimm. ${ }^{8}$ Am Ende meinte die Präsidentin des BVerfG, die Grenze zumutbarer Kritik sei erreicht. ${ }^{39}$

Was um Himmels willen hatte das BVerfG, genauer: Was hatten drei Richter und zwei Richterinnen seines Ersten Senats im Namen des Volkes entschieden? Nicht "Kruzifixe verfassungswidrig", wic die Frankfurter Allgemeine Zcitung ebenso knapp wic falsch meintc. Wohl aber, daß es dem Landesgeserzgeber von Verfassungs wegen verwehrt sei, das Aufhängen von Kruzifixen in den Klassenräumen öffentlicher Schulen zur Pflicht zu machen. Glaubens- und Gewissensfreiheit, immerhin das. älteste Grundrecht überhaupt und in Art. 4 I GG ohne Vorbehalt verbürgt, verböten es, einer bestimmten - hier: der katholischen - Glaubensüberzeugung "mit staatlicher Unterstützung Ausdruck zu verleihen« und dic andersgläubigen Schüler zu zwingen, "unter dem Kreuz zu lernen.$^{40}$

Wer den Blick zwischen der Verfassung einer demokratischen Republik und $\mathbb{I _ { 3 }} \mathrm{I}_{3}$ BayVSO hin- und herwandern läßt und im übrigen zu Befürchtungen, das Abendland könnc an kreuzeslosen Klassenzimmern untergehen, Abstand zu halten vermag, wird an Beschluß und Begründung wenig Sensationelles finden. Mit ( 1 ) der negativen Religionsfrciheit, (2) der Trennung von Staat und Kirche in einer säkularisierten Gesellschaft und dem (3) daraus sich ergebenden Prinzip der weltanschaulichen Nichtidentifikation sowie (4) dem Grundsatz der Parität von Kirchen und Bekenntnissen sind die verfassungsrechtlichen Markierungspunkte vorgezeichnet. Der staatliche Schulherr hat sie bei der Veranstaltung von Schule zu respektieren. Regionale Traditionen oder Erzichungsziele in Landesverfassungen, wie die bayerische Ehrfurcht vor Gott (Art. ${ }_{3}$ I II BV), erlangen auch im Durchgang durch das föderalistische Prinzip nicht die Kraft, bundesverfassungsgesetzlich verbürgte Grundrechte einzuschränken.4" $\mathrm{Da}$ diese, wenn ihre Verletzung festgestellt werden kann, legislativen Mehrheiten in den Arm fallen, also minderheitenschützend wirken, liegt nicht an Karlsruhe, sondern der Struktur solcher Verbürgungen als negativen Kompetenzbestimmungen. ${ }^{4 z}$

Kann mithin das von der Mehrheit des Ersten Senats befürwortcte Ergcbnis schwerlich als anstößig bezeichnct werden, so gibt noch weniger deren Sprache Anlaß zur Beanstandung. Freilich macht die Senatsmehrheit unverblümt und (für viele schwer erträglich) unmißverständlich deutlich, daß das Kreuz nicht zugleich für die einen

I. 9. 1995, der die „Ruckkehr zur Gesınnungstaterschaft* befurchtet. Ausfuhrlıcher: Ders., Die ungeliebte Legalitat, in: Merkur I995, $1084 \mathrm{ff}$. (interessant ist die Interpretation des Kruzifix-Streits als Fortsetzung der Brent-Spar-Hysterie) und Herz, Reaktionare Vorstellungen, in: Merkur 1995, $1141 \mathrm{ff}$. Kutscha, Gotterdammerung in Karlsruhe, in: Blatter fur deutsche u. internat. Politik, H. 10 (1995), diagnostiziert ein nbigottes Verhaltnis zum Rechtsstaat* und Akzeptanzprobleme.

36 Hierzu auch Czermak, NJW 1995, 3348 ff. In kritıscher Distanz zur Senatsmchrhet, hinsichtlich Art. 4 I GG jedoch alles andere als uberzeugend: Link, NJW $1995,3353 \mathrm{ff}$.

37 Presseerkl. des Vizeprasidenten des Ersten Senats, FR v. 12.8. 1995. Der 1. Leitsatz lautet: „Die Anbringung eines Kreuzes oder Kruzifixes in den Unterrschtsraumen einer staatlichen Pflichtschule, die keine Bekenntmisschule ist, verstoßt gegen Art. 4 Abs. I GG. . Die Korrektur prazisıerte, wie aus den Beschlußgrunden ersichilich, daß nur die staatlich angeordnete* Anbringung verfassungswidng ist (EuGRZ 1995, 359).

38 Grimm, Unter dem Gesetz, FAZ v. 7.9.1995

39 Limbach, „Die Grenzen sind erreicbta (Interview), Der Spregel Nr. 35 (1995), $34 \mathrm{ff}$

40 EuGRZ 1995, 363.

41 Das scheinen die dissentierenden Richter zu ubersehen (EuGRZ 1995, $366 \mathrm{ff}$ ).

42 Ausführlich dazu Denninger, Der Einzelne und das allgemene Gesetz, KJ 1995, 425 ff. Eben das verkenss Link, NJW 1995, 3348 if. 
und in einem Kontexc als christliches Symbol, für die anderen und in anderem Kontext als nur kulturelle Errungenschaft und Verkörperung abendländischer Werte herhalten kann. ${ }^{43}$ Schlicßlich fehlt auch der besonders häufig attackicrten Metapher "unter dem Kreuz lernen "jeder Überraschungseffekt. Daß "unter dem Kreuz" Handlungen von verfassungsrechtlichem Belang geschehen können, stellte das BVerfG bereits langc zuvor in seiner Entscheidung zu Kreuzen in Gerichtssälen fest. ${ }^{44}$ Es kann also keine Rede davon sein, die Kruzifix-Mehrheit des Ersten Senats habe "mit der Brechstange argumentiert «, 45 wie ein ehemaliger Verfassungsrichter meinte kundtun zu müssen. Im Gegenteil ist jenem BVerfG, das unter aktiver Mitwirkung eben dieses Kritikers die Vor-Urteile zum Kruzifix-Beschluß fällte, vorzuwerfen, daß es in puncto "christlicher Gemeinschafts(?)schule « und Schulgebet nicht den Mut hatte, dic Säkularisierungsgewinne des GG argumentativ einzulösen, statt dessen ein Exerzitium zur Unbestimmthcit des Rechts ${ }^{16}$ vorführte, dem dic Senatsminderheit in ihrem abweichenden Kruzifix-Votum nun freudig nacheifert.

Aus dem engeren Kontext des Kruzifix-Bcschlusses ist nicht recht zu crsehen, was genau es war, das die Deiche der (wohl überwiegend geheuchelten) Empörung anstach. Dem Bruch mit der Rechtsprechung zu Schulgebet und Gemcinschaftsschulen, wiewohl beachtlich, fehlt für sich genommen die hinreichende Brisanz. Auch wegen dicses Bruchs nunmehr befürchtete Einflußverluste der Kirchen erklären nicht angemessen das teilweise hysterische Gezeter über die möglicherweise krcuzesfrcie Zone Schule. Daß Politiker mit rechtspopulistischen Neigungen, denen die ganze Richtung des Ersten Senats oder das Verfassungsgericht als Kontrolleur nicht paßt, als Trittbrettfahrer des Kreuzzuges gegen Karlsruhe auf billige Zustimmung aus waren und zugleich dem BVerfG vor anderen "allzu liberalen «, sprich: grundrechtsfreundlichen Sprüchen - zumal in Sachen Asyl ${ }^{17}$ - den Schneid abkaufen wollten, läßt sich mit einer Reihe von Äußerungen belegen ${ }^{48}$ und führt zurück zur Frage nach der gefährdeten Autorität des Verfassungsgerichts.

43 "Das Kreuz gehort nach wie vor zu den spezifischen Glaubenssymbolen des Christentums. Es ist geradezu sein Glaubenssymbol schlechthin. Es versinnbildlıcht die im Opfertod Christi vollzogene Erlósung des Menschen von der Erbschulda (EuGRZ 1995, 364). Dagegen stellt dic dissentierende Minderheit apodikrisch die empirısch mehr als angreifbare und normativ fragwurdige Behauptung auf: „ Es mag sein, daß in einem Schuler christlichen Glaubens beum Anblıck des Kreuzes un Klassenzimmer teilweıse diejengen Vorstellungen geweckt werden, die von der Senatsmehrhest als Sinngehalt des Kreuzes geschildert werden. Fur den nichtglaubigen Schuler hingegen kann das nicht angenommen werden. Aus seincr Sicht kann das Kreuz im Klassenzımmer nicht die Bedeurung cines Symbols fur christliche Glaubensinhalte haben, sondern nur die eines Sinnbilds fur die Zielsetzung der christlichen Gemeinschaftsschule, namlıch fur die Vermirelung der Werte der abendlandischen Kultur, und daneben noch die eines Symbols einer von ihn nicht geteilten, abgelehnten und vielleicht bekámpften religiosen Uberzeugunga (EuGRZ I995, 368).

44 BVerfGE 35,336

45 Benda, einer der Autoren der Schulgebets- und Gemeinschaftsschulen-Urucile, zit, nach Fromme, Wenn ein Gericht \%uviel will (Fn. 32).

${ }_{46} 6$ Die "christliche Gemeinschaftsschule sıgnalısiert bereıts Im Begriff eıne Paradoxıe, die dem Gericht hätte auffallen mussen. Wenn in der Urteilsbegrundung (BVerfGE $41,44 \mathrm{ff} . \mathrm{u} .77 \mathrm{ff}$.) von wreligiosen. bzw, schristlichen Bezugen* die Rede ıst, so mag man diese im Hinblick auf Art. 7 III oder V GG fur verfassungskonform halten, sollte sich dann freilich uber das Konzept der „Gemeinschaft « Gedanken machen. (Krit. hierzu Renck, Aktuelle Probleme der christichen Gemeinschaftsschule, KJ 1994, $488 \mathrm{ff}$. mwNachw.) Die These, das Schulgebet als oreligioser Bekenntnisakt, das außerhalb des Religionsunterrichts gesprochen wird, ist niche Teil des allgemeinen Schulunterrichts, der im Rahmen des staatichen Bildungs- und Erziehungsauftrags ertcilt wird a (E s2, $238 \mathrm{f}$.), erscheint bedenklich genug. Jedoch die Schlußfolgerung, damit falle das Schulgebet sauch nicht unter die Vermitclung christlicher Kultur- und Bildungswertee (S. 239), 1st schlechterdings absurd. - Allgemein zur Unbestimmtheit: Frankenberg, Der Ernst im Recht, 3036 .

47 Hier ist frellich der Zweite Senat am Zug.

$48 \mathrm{Im}$ Anschluß an die Ableitung eines "Rechts auf das Kreuz fur die christliche Mehrheıt der bayir. Bevolkerung warnte der Bayer. Ministerprasident Stoiber des BVerfG davor, den Asylkompromiß auszuhebeln (Beriche der FAZ v. 9.9. 1995). Vgl, auch Knapp, Die Frau in der Zentralc ..., FK v. 16. 8. 1995 Fromme, Ein Ruf wird verspielt, FAZ v. 14.8 .1995$. 
Was gefährdet ist oder sein könnte, muß, so will es die Logik, zunächst einmal existieren. $\mathbb{\$} 3$ r BVerfGG gibt einen Hinweis auf die Autorität des BVerfG: Seine Entscheidungen haben institutionellc Folgen. Als wichtigste wird dort die Verbindlichkeit genannt. \$3s BVerfGG ergänzt diese um den Gesichtspunkt der Vollstrekkung. Von den Verfassungsgenossinnen und -genossen sowie vor allem von den anderen öffentlichen Gewalten wird Gehorsam erwartet. Deshalb fällt hinhaltender Widerstand des Gesetzgebers, der Aufträge aus Karlsruhe nicht erfüllt, ${ }^{49}$ unangenehm auf. Desgleichen Unbotmäßigkeit aus den Reihen der Justiz ${ }^{50}$, erst rech maskierter oder gar offener Ungehorsam von Sciten der Exekurives'. Selbst übermäBig scharfe Urteilsschelte aus der Mitte des souveränen Volkes ${ }^{52}$ gilt als unfein. Woraus zu schlicßen ist: Das BVcrfG nimmt im politischen System der Bundesrepublik eine hervorgchobene Stellung ein, seine Richterinnen und Richter genießen eine besondere Autorität. 53

Gleich hinter "Autopsie « definiert der Große Duden, Autorität sei der "auf Leistung oder Tradition bcrukende maßgebende Einfluß und das daraus crwachsende Ansehen und die maßgebende Person selbst; in der Theologie die nicht einsehbarc, Glauben und Gehorsam fordernde Macht «. ${ }^{34}$ Das Wörterbuch unterscheidet ferner: "autoritär« - in illegtimer Autoritätsanmaßung handelnd - und »autoritativ« - auf echte Autorität gestützt, in legitimer Vollmacht handelnd, maßgebend, entscheidend. Kürzer ließc sich sagen: Autorität hat, wer erwarten kann. Genauer: wer erwarten kann, daß die mit dem Anspruch auf Verbindlichkeit gesetzren Zeichen freiwillige Nachachtung finden. Die Freiwilligkeit, die eine legitime Autorität auszeichnet, verwandelt autoritäre Herrschaft in autoritative Macht und einc Leistung, wie Recht sprechen oder Geserze beschließen und diese ausführen, in eine Personen oder Institutionen zugeschriebene Qualität. Sic besitzen Legitimität. Auf Freirvilligkeit sind Verfassungsgerichte in besonderer Weise angewiesen, da ihnen zur Durchsetzung ihrer Entscheidungen keine Zwangsgewalt zur Verfügung steht.

Folgen wir den Idealtypen legitimer Autorität Max Webers, so scheiden Charisma und Tradition vorderhand als Autoritäts- bzw. Legitimitätsquellen aus. Die These

49 Vgl. BVerfGE $8,210 / 216 ; 17,148 / 155 ; 22,163 / 172$ und $25,167 \mathrm{ff}$., hunsichtheh der Gleichstellung nichtehelicher Kinder. Zu neueren nicht erledigten Auftragen an den Gesetzgeber vgl. Salgo, KritV 1994, $262 \mathrm{ff}$.

so Gezugelten justiziellen Ungehorsam demonstrierte der BGH (NJW 1988, $173^{8)}$ gegenuber der Mutlangen-Entscheidung des BVerfG (E 73, 206), als es die verfassungsgerichtlich zugelassene Berucksichtigung der Fernziele im Rahmen der Verwerflichkeit nunmehr in die Strafzumessung verbannte. Vgl. auch die BVerfG-Rige des OLG Nurnberg, es habe sich snicht mit der gebotenen Nuehternheit mit seiner Rechtsprechung auseinandergesetzta ( $2 \mathrm{BvR} 219 / 94)$.

st Wahrend die Krtik mißliebiger Urteile durch Regierungsmitglieder gang und gabe ıst und diese dabeı nicht immer Maß halten (siehe etwa die Reaktionen auf den sSoldaten $\alpha$-Beschluß, auf die jünste Sitzblockaden-Entscheidung und zuletzt das Spionage-Urteil), durfte offener exekutiver Widerstand die Ausnahme sein. Als eine solche gilt der Hinweis der Bundesregierung an den Prasidenten des BVerfG aus Anlaß der Schleyer-Entscheidung ( $\left.E_{4} 6,160\right)$, man werde sıch auch einer verfassungsnchterlich verfugten Freilassung der inhaftierten Terroristen widersetzen.

\$2 Für scharfe, Souveranitatsmangel dokumentierende Angriffe auf das BVerfG zur neuesten SitzblockadenEntscheidung (NJW 1995, 1141) und zum "Soldatene-Beschluß (NJW 1994, 2943) vgl. Ruchers, Als Motor des Zeitgeistes ungeeignet, und die eher gehassigen, auf Mißverstandigung angelegten Ausfalle von Roelleeke, B10-Recht oder die Sanftmut von Gesaß-Protestierern, in: NJW 1995, is 2 sff.

S3 Zum Begriff der Autoritat vgl. Arendt, Was ist Autoritit?, in: dies., Fragwurdıge Traditıonsbestande im politischen Denken der Gegenwart (Frankfurt/M. o. J.), 117 ff.; Friedrich, Politische Autoritat und Demokratie, in: ZfPol NF 7 (1960), iff.; Horkheimer $(\mathrm{Hg}$.$) , Studien uber Autoritat und Familie (Paris$ 1936), insbcs. $3 \mathrm{ff}$. (Horkhemer, Theoretısche Entwurfe) und $136 \mathrm{ff}$. (H. Marcuse, Ideengeschichte); Sennett, Autoritzis (Frankfurr/M. 198s) und Max Weber, Wurtschaft und Gesellschaft, s. Aufl. (Tübungen igio), $122 \mathrm{fl}$. und $551 \mathrm{fl}$.

S4 Fremdworterbuch, 2. Aufl. (1971), 82. 
eincr "außeralltäglichen Hingabe an die Heiligkeit, Heldenkraft oder Vorbildlichkeir«s' der Karlsruher Richterinnen und Richter wäre allzu ironisch - ein Ton, der auch von ihnen nicht sonderlich geschärzt wird. ${ }^{56}$ Ebensowenig können wir hier das Werk eines "Alltagsglaubens an die Heiligkeit von je her geltender Traditionen" vermuten. ${ }^{57}$ Das Entzauberungswerk der Aufklärung hat ein für allemal den unbeschwerten Rückgriff auf eine ehrwürdigc, unbezweifelbare Überlieferung verlegt. Im Kontext einer säkularisierten Gesellschaft müßtc das oberste Gericht seine Traditionsbasis wohl oder übel mit posttraditionalen Mitteln konstruieren. Diese paradoxe Aufgabe zu lösen, ist ihm weder bisher geglückt noch in Kontext einer demokratischen Republik zuzumuten..$^{58}$ Davon abgesehen stehen das jugendliche Alter der Institution sowie die unabschließbare Grundsatzdebattc über ihre demokratische Legitimität, auf dic sogleich zurückzukommen sein wird, jener für dic Geburt und Entwicklung einer Tradition crforderlichen, weihevollen Ruhe entgegen.

Also bliebe, wenn Weber das letzte Wort hätte, nur der "Glaube an die Legalität gesatzter Ordnungen und des Anweisungsrechts der durch sie zur Ausübung von Herrschaft Bcrufenen «"'. Solche legal-rationale Autorität könnte sich hicr aus der Verfassung ableiten und in der Tat einer Institution zugeschrieben werden. Diese Autoritätsgrundlage drängt sich auf, ist jedoch so unproblematisch nicht.

Am Anfang steht der Text: das Grundgesetz. Es überantwortet unter anderem dem BVcrfG "rechtsprechende Gewalt " (Art. 92) und weist diesem bestimmte Entscheidungsverfahren zu (Art. 93), die auf entsprechendc Befugnisse schließen lassen. Allerdings faßt sich das GG kurz hinsichtlich der Mitglieder des Gerichts und ihrer Wahl, legt sodann die Ausgestaltung der Gerichtsverfassung nebst Verfahrensordnung in die Hände des Gesetzgebers (Art. 94). Dcr vom Grundgesetz informierte Blick entdeckt ferner dic Bindung auch des Verfassungsgerichts an die Grundrechte (Art. I III) und an "Gesetz und Rechr " (Art. 20 III). All das - die Ermächtigung des BVerfG durch die Verfassung - durchaus prima facie eine Grundlage für einen Glauben an die Autorität kraft gesetzter Ordnung.

Freilich: Bei Lichte besehen, offenbart dicse Grundlage tiefe Risse und Spannungen, die an ihrer Tragfähigkeit Zweifel aufkommen lassen. Diese konzentrieren sich, geweckt durch dic beachtliche Machtfülle des BVerfG, inshcsondere sein Recht, Beschlüsse des Gesetzgebers zu prüfen und bei Verstößen gegen das GG zu verwerfen, zunächst auf eine präzise Positionsbestimmung des Gerichts in Verfassungsgefüge. Zumal die in Art. 93 I Nr. I -4 und 100 I GG vorgesehenen Verfahren legen nahe, in Karlsruhe ein "oberstes Verfassungsorgan* zu vermuten. Diese Stcllung wird ihm jedoch von den Interpretationseliten verwehrt. Nach sherrschender Meinunga. Mangels Verfahrensautonomie bleibt dem BVerfG demnach nur der Verlegenhcitsstatus cincr "verfassungsorganähnlichen * Institution. ${ }^{60}$ Und doch soll cs

\footnotetext{
ss $M$. Weber, Wirtschaft und Gesellschaft, 124.

s6 Vgl. BVerfG - 2 BvR 291/94.

57 M. Weber, Wirtschaft und Gesellschaft, 124

s8 Selbst wenn die Richter immer weder versucht haben, ihren Entscheidungsgrunden die Rückendeckung einer fragwurdigen Tradituon zu besorgen. Zur Argumentationsfigur der Tradition in der Rspr. des BVerfG vgl. die materıal- und gedankenreiche Monographic von Blankenagei, Tradition und Verfassung. Neue Rechtsprechung und alte Geschichte in der Rechtsprechung des Bundesverfassungsgerichts (Baden-Baden 1987).

59 Weber, Wirtschaft und Gesellschaft, 124

6o Zum Streıt uber dıe Organqualitat vgl. Schlarch, VVDStRL 39 (1981), 133; Ipsen, Stastsrecht I, 6. Aufl. (1994) Rn. 845 ff.; Stern, Staatsrecht II, 345 mwNachw, und Pestalozza, Verfassungsprozeßrecht 3. Aufl,

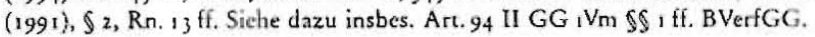


dieser Institution selbst geschaut und ausgedeutet werden kann, an einer strikten Bindung an das Prinzip demokratischer Legalität fehlen. Zum anderen durchzieht seinc Entscheidungspraxis cin höchst eigenwilliges Verständnis von und Verhältnis zu richterlicher Zurückhaltung. ${ }^{76}$ Wertordnungslehre als potentielle Bevormundung des Volkes und seiner öffentlichen Gewalten sowie wiederholte Belehrungen des Gesetzgebers über die Prinzipien aktiver Sozialgestaltung rücken das BVerfG in die gefährliche Nähe einer sich über das Verfassungsgesetz crhebenden Letztentscheidungsinstanz.

Wir können bilanzicren: Autorität kraft göttlicher Gnade, offenbarter Wahrheit oder chrwürdiger Tradition kommt in einer demokratischen Republik nicht in Frage. Autorität kraft Verfassungslegalität ist von Paradoxien nicht frei. Autorität durch Leistung wäre eher denkbar, aber - angesichts der, wie oben illustriert, mal von Staats-, mal von Grundrechtsfreunden vorgetragenen Urteilsschelte - höchst prekär. Mithin spricht alles für eine paradoxe Intervention. In diesem Sinne soll hier die These verteidigt werden, daß das BVerfG seine Autorität dem Konflikt verdankt. Genauer: der selbstreflexiven Wahrnehmung gesellschaftlicher Kontroversen auf der Ebene und in der Sprache der Verfassung als Ausdruck einer "grundlegenden Konvention ".77 Mit "grundlegender Konvention « werden jene Konfliktregeln bezeichnet, die sich einer expliziten oder stillschweigenden, allerdings nur wirksamen, wenn in der sozialen Praxis der Zivilgesellschaft aufweisbaren Übereinkunft verdanken. Als grundlegend gelten in diesem Kontext jene für eine einigermaßen zivilisierte Austragung von sozialen Kämpfen unverzichtbaren Regeln, insbesondere die Respektierung der physischen und psychischen Integrität der Konfliktgegner sowie die Anerkennung ihrer gleichen Freiheit. Dic Bezeichnung "Konvention « soll die Möglichkeit der Änderung und die Notwendigkeit der Aktualisierung, damit zugleich die Risiken einer solchen Übereinkunft hervorheben. Hiermit treten die Mitglieder einer Zivilgesellschaft als Akteure ins Bild, die als einzelne und in Assoziationen die verschiedenen Öffentlichkeiten »bevölkern « und dort ihre unterschiedlichen Bedürfnisse und Interessen, Überzeugungen und Forderungen zur Geltung bringen. ${ }^{8}$

Erstens nimmt das BVerfG soziale Konflikte gleichsam von außen als Beobachtungsund Schlichtungsinstanz wahr. Und zwar als das legitime, auf Dauer gestellte Aufeinanderprallen kontroverser Meinungen und Forderungen, Lebenspläne und Weltbilder. Das Gericht befindet sich damit auf Augenhöhe einer interessengespaltenen, radikal pluralistischen Gesellschaft. Frühzeitig erkannte es, daß Konflikte für eine demokratische Republik konstitutiv und folglich Dreh- und Angelpunkt für die Auslegung der Freiheiten politischer Kommunikation sind..$^{79}$ Von Lüth ${ }^{80}$ bis Brok-

ders., Der gebandigte Leviathan, 143 ff.; Preuß, Internalısierung des Subjekts, 261 ff.; ders., Legalitat und Pluralismus, $22 \mathrm{ff}$

76 Vgl. BVerfGE 2, 79/96; 64, 158/168f.; 66, 84/95 (Zuruckhaltung gegenuber dem Gesetzgeher); dagegen 35,$1 ; 39,1$ und $25,167 / 178 \mathrm{ff}$. (wenig Zuruckhaltung gegenuber dem Gesetzgeber); $18,224 / 240$; 54 , $208 / 215$ und 66,116 (Zuruckhaltung gegenuber der Just1z), dagegen $70,93 / 97$ und $80,48 / 51$ (weniger Zuruckhaltung gegenuber der Justiz).

77 Diese ogrundlegende Konvention* mag auch als demokratische Streitkultur bezeichnet werden. Ausfuhrlich dazu Frankenberg, Die Verfassung der Republik. Autoritat und Solidaritat in der Zivilgesellschaft (1996), bes. i gof. und 19 iff.

78 Ausfuhrlich hierzu Frankenberg (Fn. 77), Kap. II und Rodel/Dubiel/Frankenberg, Die demokratische Frage (Frankfurt 1989).

79 Am pragnantesten wohl im Luth-Urteil: - Das Grundrecht auf freie Meınungsaußerung ist als unmittelbarster Ausdruck der menschlichen Personlichkeit eines der vornehmsten Menschenrechte ubcrhaupt ... Für eine freiheitlich-demok ratische Staatsordnung ist es schlechthin konsutuerend, denn es ermoglicht erst die standige geistige Auseinandersetzung, den Kampf der Meinungen, der ihr Lebensel' 'inent ist." (BVerfGE 7, 198/208; vgl. auch BVerfGE s, 8s/30s)

80 BVerfGE 7,198 . 
dorf ${ }^{\beta 1}$ haben die Richterinnen und Richter - sukzessive, wenngleich nicht immer gradlinig - ihr Konfliktbild vom Modell des quietistischen, am idealisierten Marktgeschehen orientierten, "rein geistigen Wettstreits* der Meinungen abgelöst und mit aller geborenen Vorsicht - den "Kampl der Meinungen ${ }^{\$ 2}$ bis zur Grenze "Schmähkritik ${ }^{83}$ zugelassen. Selbst nicht-argumentarive, "nötigende Einwirkungen « ${ }^{8 \triangleleft}$ auf andere stellte das BVerfG unter den Schurz der Verfassung und bescheinigre dem zivilen Ungehorsam immerhin die Dignität eines diskussionswürdigen Phänomens, um diese Protestform schließlich realitätsnah aus dem Bannkreis der Gewaltnörigung zu befreien. ${ }^{\text {s }}$

Mit solchen Entscheidungen hat das BVerfG, ob bewußt oder nicht, jene Ressource gehegr, von der eine Institution zehren muß, die den Gehorsam oberster Verfassungsorgane $e^{86}$ notfalls weder mit polizeilichen Mitteln erzwingen noch mit Geld erkaufen kann. Diese Ressource ist die kritische Aufmerksamkeit und politische Handlungsfähigkeit der Akteure in den verschiedenen politischen Öffentlichkeiten, die die Vitalität einer Zivilgcsellschaft konstituieren. Daß diese Öffentlichkeiten unberechenbar, fragmentiert, in Staats- und Grundrechtsfreunde, in Gegner und Befürworter der Verfassungsgerichrsbarkeit gespalten sind, tut ihrer Bedeutung als Autoritätsressource keinen Abbruch. Im Gegenteil: Von einer reinen Akklamationsöffentlichkeit hätre das Verfassungsgcricht bereits auf mittlere Sicht keine Autoritätsimpulse zu erwarten. Erst öffentliche Kontroversen erzeugen von Fall zu Fall jene Reibungsenergien, für deren temporäre Entladung und Ableitung sich eine Verfassung als geeignete und erforderliche Schiedsinstanz empfehlon und erweisen kann.

Zweitens betciligt sich das BVerfG, durchaus ambivalent, selbst an politischen Konflikten. Nicht mehr nur Forum oder Bühne für andere, wagt es sich nunmehr selbst in die öffentliche Arena, gibr seine üblicherweise als Neutralität deklarierte Zurückhaltung auf und wird aktiv. Weniger bei der Frage, ob der Bund das Schornstcinfegerwesen ordnen dürfe ${ }^{87}$ oder wie das Nettoentgelt eines einer Kirchensteuer crhebenden Kirche nicht angehörenden Arbeitslosen zu berechnen sei ${ }^{88}$. Wohl aber stets dann, wenn es die ohnehin nicht immer sichere verfassungsrechtliche Deckung verläßt und sich relativ unverhüllt mit den öffentlichen Gewalten ${ }^{k,}$ oder gar dem mutmaßlichen Volkssouverän, jedenfalls einer Mehrheitsmeinung, ${ }^{90}$ anlegt. In diesen Fällen dekonstruiert das Gericht - cher unfreiwillig - jene mic der Vorstellung einer Schiedsinstanz verbundene Doktrin, seine Funktion sei, Frieden zu stiften, und prä-

81 BVerfGE 69, 315/344 ff.

82 BVerfGE 7, 198/208. Vgl. die vorsichtugere Formulierung in BVerfGE 2s, 256/264 (Blinkfuer) und BVerfG NJW $1989,381 / 382$.

$83 \mathrm{Vgl}$. BVerfGE 60,234/240;62,230/244f. Vgl. dazu Kubler, Wirtschaftsordnung und Meınungsfreihest. 84 BVerfGE $69,315 / 344 \mathrm{ff}$.

85 Ohne aber - richtigerweise - ein "Recht " auf zivilen Ungchorsam zu verkunden. Vgl. BVerfGE 73, 206 und st.Rspr. bis zur Wende hinsichtlich der Beurteilung des svergestugten Gewaltbegriffse als nicht vereinbar mit Art. 103 II GG in BVerfG NJW 1995, 1141.

$86 \mathrm{Im}$ ubngen ist das BVerfG hinsichtlich der Durchsetzung seiner Urtele so machtlos nicht; vgl. etwa \$35 BVerfGG und BVerfGE 6, 300 (Vollstreckung des KPD-Verbors).

8) BVerfGE $1,264$.

88 BVerfGE $90,226$.

89 Ein solcher Fall war die Entscheidung zu sSoldaten sind Morders, Nach den scharfen Reaktionen vor allem der Exekutive auf freisprechende Urteile der Strafgerichte - erwartbar die Finporung des Bundesministers fur Verteidigung, uberraschend die Krıtuk des vormalıgen Bundesprasidenten - hatte der vorsichtige Kammerbeschluß, der diese provokante Außerung in den Schurzberesch von Art. s I GC einbezog, zwangslaufig den Charakter einer Parteinahme. Vgl. BVerfG NJW 1994, 2943 und die Enlassungen oder eigentlich: Auslassungen von Ruthers und Roellecke (Fn. 4) und dic Kommentare von Herdegen, NJW 1994, 2933 ff.; L.orenz NJ 1994, 56 Iff. und Sochring NJW 1994, $2926 \mathrm{ff}$.

90 In der Entscheidung zur Fristenlosung fuhrte das BVerfG aus, daß es sich auch von einem sallgemennen Wandel der hieruber in der Bevolkerung herrschenden Anschauungen. nicht irritieren lassen konnc; BVerfGE $39,1 / 67$. 
sentiert sich selbst als politischer Akteur. Dieser Rollenwechsel ist prekär, was sich beispielsweise dann zeigt, wenn das Gericht aus einer die Verfassung überwölbenden, vermeintlich "objekriven Wertordnung " eine argumentative Waffe für sich selbst schmiedet, oder wenn es jenseits des Horizonts einer strict interpretation Verfassungsaufträge auffindet und an die öffentlichen Gewalten adressiert.

Gelingen kann und innerhalb des Rahmens einer demokratischen Republik zu halten ist ein solchcr Rollenwechsel freilich nur unter der teilnehmenden Beobachtung und notfalls protestierenden Mitwirkung einer einigermaßen wachen und kritischen Öffentlichkeit. Fällt diese aus, kann also von einer Zivilgesellschaft keine Rede sein, dann können sich in der Tat die von den Gegnern der Verfassungsgerichtsbarkeit betürchteten Gefahren cinstellen. Hält jene jedoch die Augen offen, und ist sie zum wrole-taking « bereit, d.h. fähig und willons, für die "grundlegendc Konvention " einzutreten, dann vermag sie als "multikulturelle Gesellschaft der Verfassungsinterpretcn« die für eine zivile Regelung gescllschaftlicher Konflikte notwendigen Prinzipien zu verteidigen. ${ }^{9+}$

Drittens spiegelt das BVerfG das, was es wahrnimmt, nämlich die Konflikthaftigkeit der Gesellschaft, in seiner eigenen Entscheidungs- und Begründungspraxis wider. Allerdings mit der berufs- und situationsbedingten Behutsamkeit. Richterliche Urteilc, das lernt man im juristischen Studium oder in der Referendarzeit - oder nie -, sind so zu begründen, daß der Zweifcl schweigt. Deshalb beraten Gerichte ihre Sprüche unter Ausschluß der Öffentlichkcit und bringen ihre Gründe mit Hilfe der Relationstechnik auf eine Stromlinie, die zur »objektiven Wahrheit « oder authentischen Interpretation, zur offensichtlichen (Un-)Begründetheit einer Klage oder ähnlichen Eindeutigkeiten führen soll.

Dieser Methode, dic alle Einwände zu Grabe tragen, jedenfalls einschläfern will, folgen die Mitglieder des BVerfG nur aut halbem Wege. Nach ihrer geheimen Beratung gewährt ihnen das Gesetz die Freiheit zu offenem Dissens ( $\$$ 30 II BVerfGG), wobei die Dissidenten entweder anonym bleiben ${ }^{92}$ oder in einem Sondervocum in Erscheinung treten können. ${ }^{93}$ Wennglcich dic Institution ihr Personal naturgemäß mchr oder weniger schwach nötigt, nach außen mit einer Stimme zu sprechen, fehlt es niche an abweichenden Voten, dic häufig mehr Einfluß gehabt und mehr für das Ansehen des BVerfG getan haben als die jeweilige Mehrheitsmeinung. ${ }^{94}$ Wichtiger noch: Die Zulassung von offenem Dissens führt eine Transparenz ein, die nicht nur der Architcktur scines Sitzes, sondern einem demokratisch-republikanischen Stil cher entspricht als demonstrative Geschlossenheit. ${ }^{95}$ Dieser Stil ebenso wic ihre Doppelrolle legen den Verfassungsrichterinnen und -richtern nahe, Abschied zu nehmen von einem Selbstbild als »Knecht des Rechts", das sich mit Formeln wie "Distanz und Diskretion", "Dienst am Recht" und dem "Verzicht auf politische

91 Der zivile Ungehorsam (im strıkten Sınne) laßt sıch als ein solches role-taking interpretieren. Zur Phanomenologie und normatıven Bedeutung dieser Protestorm vgl. Frankenberg, Ziviler Ungehorsam und rechtsstaatliche Demokratie, JZ 1984 und ders., Der zivile Ungehorsam auf dem Rechtsweg, in: Roth/Ruche (Hg.), Neue sozıale Bewegungen in der Bundesrepublik, 2. Aufl. (1991).

92 Vgl. etwa die $4: 4$ Konstellation in der ersten und die $s: 3$ Konstellation in der zweiten SitzblockadenEntscheidung (BVerfGE 73, 206 und BVerfG NJW 1995, 1114).

93 Zur Geschichte und Praxıs der Sondervoten: Lamprecht, Richter contra Richter. Abweichende Meınungen und ihre Bedeutung fur die Rechtskultur (Baden-Baden 1992).

94 Solche Ehren- und Ansehensrettungen waren - nie unstreıug - die Voten der Dissidenten und Dissidentunnen zum Abhor-Urtell (E 30, 1/33 ff.), zu den Entscheidungen betreffend die Fristenlosung (39, 1/68 ff.), die Treuepflıcht ( $E_{3}, 378$ ff.), die Parteienfınanzierung ( $\mathrm{E}_{73}, 40 / 103 \mathrm{ff}$.), die parlam. Kontrolle der Geheimdienste (E $70,324 / 366$ ff.), die Neuordnung des Rechts der Kriegsdienstverwengerung (E 69, $1 / s 7 \mathrm{ff}$.) und uher Strafbarkett des Umgangs mit Cannabis-Produkten (E 90, $145 / 212$ ff.). Vgl. dazu auch Lamprecht, Ruchter contra Richter (Fn. 93).

95 Daß sıch die Installation des Dissenses auch funktionalıstisch interpretieren laßt - crwa nach Maßgabe von Luhmann, Legitımation durch Verfahren (Neuwied/Berlin 1969) -, liegt auf der Hand. 
Ambitionen " in einem "entsagungsvolle(n) Amt ${ }^{96}$ noch angemessen beschreiben läßt. Die Entsagungen waren vorgezeichnet durch die Maxime, nach der ein Verfassungsrichter nicht vertcidigt, nicht kommentiert und nicht sauf Angriffe, Kritik und Unverstand " reagiert. ${ }^{97}$ Im Kruzifix-Konflikt - freilich nicht erst hier ${ }^{99}$ - demonstrierten die Angegriffenen ein deutlich anderes Selbstverständnis und Selbstbewußtsein: Verfassungsrichterinnen und -richter lassen sich auf öffentliche Kommunikation ein. ${ }^{99}$ Wer das als "Entscheidungs-Marketing " kritisiert, muß sich fragen lassen, ob solchc Kritik nicht die selbstexplikative Kraft von Urteilsgründen überschätzt, heimlich noch immer der Sehnsucht nachgibt, der Zweifel möge schweigen, oder aber sich dagegen sperrt, das BVerfG in die nüchterne Realität einer demokratischen Republik einzufügen.

\section{Schlußbemerkung}

Das auf die Legitimität des Konflikts gegründete Ansehen des BVerfG, obwohl von Ambivalenzen nicht frei, hat einiges für sich. Der permanente Rollenwechsel sowie die veröffentlichten internen Differenzen arbeiten beharrlich der verbreiteten Sehnsucht nach einer quasi-transzendenten Instanz entgegen, die ein für allemal das letzte Wort hat. Mit 4:4- oder 5:3-Entscheidungen, Kursänderungen, overrulings und Grenzüberschreitungen demystifiziert ein Verfassungsgericht sich selbst und warnt zugleich vor einer Überschätzung des Legalen. ${ }^{100}$ Eine derart kommunikative und streitbare Entscheidungspraxis legt uns nahe, Abstand zu halten von Dämonisierungen oder Idcalisicrungen dieser Institution. Als Hüter und Streiter zugleich bleiben Richterinnen und Richter aus Karlsruhe auf dem Teppich einer Konflikt- bzw. Zivilgesellschaft. Sie bekräftigen die Legitimität des unabschließbaren Diskurses über dic Legitimität. Selbstreflexiv oder, wie es noch vor dreißig Jahren geheißen hättc, dialektisch.

96 So der ehemalige Verfassungsrichter Geıger, zit. nach Lamprecht/Malanowski, Richter machen Politık (Frankfurt/M. 1979), 9.

97 Geiger (Fn.96).

98 Geiger predigte offentlich Wasser und trank heinlıch Weın: Er selbst publizierte seinen Dissens mut der Richtermehrheit in Sachen EVG-Vertrag. Vgl. JZ 1953, 39 und Lietzmann, Das Bundesverfassungsgericht, 103 . Auch andere Mitglieder des BVerfG haben sich nicht davon abhalten lassen, Entscheidungen zu erlautern oder zu verteidigen.

99 Vgl. nur Grimm, Unter dem Gesetz (Fn. 38) und Lımbach, Die Grenzen sind erreicht (Fn. 39).

100 Vgl. Ehrlich, Grundlegung der Soziologie des Rechts, 3. Aufl. (1967), Vorrede. 